\title{
How patch configuration affects the impact of disturbances on metapopulation persistence
}

\author{
Séverine Vuilleumier ${ }^{\mathrm{a}, *}$, Chris Wilcox ${ }^{\mathrm{a}}$, Benjamin J. Cairns ${ }^{\mathrm{b}}$, Hugh P. Possingham ${ }^{\mathrm{a}, \mathrm{b}}$ \\ ${ }^{a}$ The Ecology Centre, The University of Queensland, Brisbane, QLD 4072, Australia \\ ${ }^{\mathrm{b}}$ Department of Mathematics, The University of Queensland, Brisbane, QLD 4072, Australia
}

Received 11 September 2006

Available online 15 November 2006

\begin{abstract}
Disturbances affect metapopulations directly through reductions in population size and indirectly through habitat modification. We consider how metapopulation persistence is affected by different disturbance regimes and the way in which disturbances spread, when metapopulations are compact or elongated, using a stochastic spatially explicit model which includes metapopulation and habitat dynamics. We discover that the risk of population extinction is larger for spatially aggregated disturbances than for spatially random disturbances. By changing the spatial configuration of the patches in the system-leading to different proportions of edge and interior patches - we demonstrate that the probability of metapopulation extinction is smaller when the metapopulation is more compact. Both of these results become more pronounced when colonization connectivity decreases. Our results have important management implication as edge patches, which are invariably considered to be less important, may play an important role as disturbance refugia.

(C) 2006 Elsevier Inc. All rights reserved.
\end{abstract}

Keywords: Disturbance; Metapopulation persistence; Patch lifetime; Spatially explicit modeling; Simulation; Patch configuration

\section{Introduction}

Classical metapopulation models ignore habitat dynamics, assuming that metapopulations are driven by extinction and colonization processes in an invariant landscape. However, empirical evidence suggests habitat dynamics are likely to play a more important role than previously appreciated. Indeed, disturbances, like fire and logging, have been shown to cause local extinctions of some species (reviewed in Gurevitch and Padilla, 2004). For instance, nearly all extinctions of British butterflies can be attributed to conversion of habitat to an unsuitable state (e.g. agricultural production or to a different vegetation type), not just to classic colonization-extinction dynamics (Thomas, 1994). In fact, the persistence of species that use early or mid-successional habitats (e.g. Wahlberg et al.,

\footnotetext{
*Corresponding author. Present address: Laboratory of Ecological Systems, Swiss Federal Institute of Technology of Lausanne (EPFL), 1015 Lausanne-CH, Switzerland. Fax: + 41416933913.

E-mail addresses: severine.vuilleumier@epfl.ch (S. Vuilleumier), chris.wilcox@csiro.au (C. Wilcox), ben.cairns@bristol.ac.uk (B.J. Cairns), h.possingham@uq.edu.au (H.P. Possingham).
}

2002), or those that have negative impacts on their own habitat (e.g. Fryxell, 2001), appear to be driven largely by extrinsic habitat dynamics, as opposed to intrinsic colonization and extinction processes. Disturbances that make patches unsuitable directly decrease metapopulation persistence by increasing the rate of local extinction, and indirectly by reducing the pool of empty patches that could be colonized.

The importance of interactions between metapopulation dynamics and patch dynamics in determining the persistence of a metapopulation is attracting increasing attention (Fahrig, 1992; Gyllenberg and Hanski, 1997; Brachet et al., 1999; Johnson, 2000; Keymer et al., 2000; Amarasekare and Possingham, 2001; Ellner and Fussmann, 2003; Thomas and Hanski, 2004; Kallimanis et al., 2005; Wilcox et al., 2006). However, many of these analyses still focus on spatially uncorrelated habitat dynamics, even though habitat disturbances are rarely randomly distributed in a landscape at the scale of most local populations. For instance, both fire and flooding are typically aggregated in time and space. In the face of such disturbances, we would expect metapopulation persistence to be influenced by 
habitat recovery and the colonization properties of the system.

The rate, amount, and temporal and spatial structure of habitat destruction play an important role for metapopulation persistence by changing patch suitability (Johnson, 2000; Keymer et al., 2000; McCarthy and Lindenmayer, 2000; Amarasekare and Possingham, 2001; Johst and Drechsler, 2003; Kallimanis et al., 2005; Wilcox et al., 2006). However, few studies have considered the impact of spatially correlated disturbances on metapopulation viability. Moreover, the interaction of these forms of disturbance and patch configuration is not presently well understood. Through its influence on patch vulnerability to disturbance and colonization events, the spatial configuration of habitat patches is expected to be important in determining metapopulation persistence. Colonization among habitat patches is more likely to occur between adjacent patches (Harrison, 1991; Doebeli and Ruxton, 1998; Thomas et al., 2001; Wiens, 2001) and in systems with more connectivity (Fahrig and Merriam, 1985; Hansson, 1991; Moilanen and Hanski, 2001). Thus, spatial configuration would affect the ability of a species to disperse and, in systems with habitat dynamics, the distance between suitable unoccupied and occupied patches (Doak et al., 1992; Diffendorfer et al., 1999). We expect that the spatial configuration of patches in a metapopulation-determined by the shape of the patch network (elongated or compact, for example) rather than the distance between neighboring patches - may affect not only colonization (Hanski and Ovaskainen, 2000) but also how much of the time a patch contains suitable habitat (Cochrane, 2003), by modifying the frequency at which patches are disturbed or colonized. If disturbances that affect habitat are spatially correlated or contagious, such as fire, hurricane, or drought, it is important to know whether some patches are less prone to such events and could provide refugia, serving as a source of colonists that might support the metapopulation following disturbance events. Specifically, there may be a correlation between patches that are well-connected and likely to be a source of colonists, and patches which are often disturbed. In this case it is unclear which patches will make the greatest contribution to metapopulation persistence. Understanding this disturbance-colonization interaction is essential from a management point of view, as it will determine which spatial configurations of patches, one of the few aspects of metapopulations that environmental planners can manipulate effectively, provide the highest metapopulation persistence.

In this paper, we analyze the effect of spatially correlated and temporally variable disturbance on patch lifetime and metapopulation persistence across different levels of subpopulation connectivity and spatial configurations of patches. We assess the effect of disturbance on metapopulation persistence in three ways. First, we compare predictions of a spatially explicit model with an analytical mean-field approximation, for the case where disturbance is spatially random. This analysis provides a verification of the simulation results and a platform for interpreting the results of more complex simulations. Second, we ask how spatially correlated disturbances affect metapopulation persistence, by examining the influence of different levels of connectivity and two extreme spatial configurations for metapopulations (elongated and compact). Third, we consider how spatial and temporal correlation in disturbance affect patch lifetime (the length of time a patch is suitable before disturbance) for the two spatial configurations, evaluating the effect of patch position in each configuration on its lifetime. Finally, we discuss the implication of our results for the management of metapopulations in dynamic landscapes.

\section{Methods}

Our spatially explicit metapopulation model is built using an objectoriented programming approach (Downing and Reed, 1996). In this model, each habitat patch is a spatial entity with a geographical position. We consider a finite metapopulation system of 100 habitat patches. In order to analyze the effects of the spatial configuration of patches on persistence we evaluate the metapopulation dynamics using two spatial configurations, a compact regular $10 \times 10$ grid of patches and an elongated regular $2 \times 50$ grid, both with one unit of distance between neighboring patches.

\subsection{Metapopulation and habitat dynamics}

The metapopulation simulation model has both habitat (or patch) dynamics and local population dynamics. Each habitat patch can be in one of the three states: (i) unsuitable and unoccupied, (ii) suitable and unoccupied, or (iii) suitable and occupied. According to its state, each patch may undergo events of recovery if unsuitable, disturbance, or colonization if suitable and unoccupied, and extinction or disturbance (and thus extinction) if suitable and occupied. The events of recovery, extinction, colonization, and disturbance each occur according to a probability of the form

$\operatorname{Pr}\{$ event $\}=1-e^{-\theta}$,

where $\theta$ corresponds to the rate of the considered event: recovery, extinction, and colonization rates ( $r, e$, and $c$, respectively). Disturbances, which affect multiple patches simultaneously, also occur according to (1) at rate $d$. However, when a disturbance occurs, the number of patches that change from suitable to unsuitable $j$ is binomially distributed as

$\operatorname{Pr}\{j\}=\left(\begin{array}{c}s \\ j\end{array}\right) p^{j}(1-p)^{s-j}$,

where $s$ is the number of suitable patches and $p$ is the per patch probability of a patch being disturbed.

Both colonization and disturbance have a spatial component to their dynamics. Colonization occurs from occupied patches to patches which are connected, suitable, and unoccupied, and is limited by a specified dispersal range (i.e. $\delta=1,2,3,4$, or $\infty$ distance units) corresponding to the degree of connection between patches. Thus for an unoccupied but suitable patch $j$, the chance it is colonized by an occupied patch $i$ is

$\operatorname{Pr}\{j$ colonized by $i\}=\left(1-e^{-c / N}\right) I\left(\delta, d_{i j}\right) O_{i}(t)$,

where $N$ is the number of patches, for any patch $i$ which is $d_{i j}$ distance units from patch $j$,

$I\left(\delta, d_{i j}\right)= \begin{cases}1 & \text { if } d_{i j} \leqslant \delta, \\ 0 & \text { otherwise, }\end{cases}$ 
and

$O_{i}(t)= \begin{cases}1 & \text { if patch } i \text { is occupied at time } t \\ 0 & \text { otherwise. }\end{cases}$

When a disturbance occurs, those patches that are affected by the disturbance are chosen either randomly or in a spatially aggregated manner. In the spatially random case, each patch is selected with equal probability. In the spatially aggregated case, those patches to be disturbed are selected by first choosing a single patch at random from the suitable patches. This patch is the first to be disturbed. Then, other suitable patches are selected to be disturbed according to a probability $p_{i}$, which depends on their distances $d_{i k}$ to the already-disturbed patches identified by index $k$ in the following equation:

$p_{i}=\frac{\sum_{k} A_{k} d_{i k}^{-2}}{\sum_{i} \sum_{k} A_{k} d_{i k}^{-2}}$,

where $A_{k}$ is equal to 1 if patch $k$ is one of those already selected to be disturbed. In both the random and spatially aggregated cases, this selection process continues until $j$ suitable patches are disturbed, where the total number of disturbed patches $j$ has the binomial distribution given by (2). This ensures that the total amount of disturbance is equal regardless of the configuration of patches.

\subsection{Simulation setup}

Metapopulation and patch dynamics are simulated as a discrete-time, sequential process of recovery, disturbance, extinction, and colonization. We assume that within a single time-step a newly occupied patch cannot colonize other patches. For each set of parameter values, at the beginning of the simulation all habitat patches are suitable and occupied, and the dynamics are then simulated for 500 time-steps. From these simulations we estimate the probability of metapopulation extinction within 500 timesteps and the mean patch lifetime as summary measures of the system dynamics. The extinction probability is defined as the ratio of the number of simulations where the metapopulation went extinct to the total number of simulations. The patch lifetime is computed dynamically; at each disturbance event, we randomly select a patch that is being disturbed and record the number of units of time since it was last disturbed. By performing a large number of simulations we construct a distribution for the patch lifetime.

To analyze the impact of spatial correlation in disturbance on metapopulation extinction we generate simulations with either spatially random or aggregated disturbances, with varying colonization rates and the following parameter values: disturbance rate $d=0.1$, recovery rate $r=0.3$, extinction rate $e=0.2$ and disturbance severity $s=0.2$. We use a Monte Carlo approach (Manly, 1991) for exploring the effect of the colonization rate on the metapopulation dynamics, randomly choosing 500 sample colonization probabilities, $\left(1-e^{-c / N}\right)$, from the range $0-0.35$. We simulated the dynamics of the metapopulation for the full 500 timesteps for each of the 500 parameter sets. We examine the effect of dispersal range, as determined by the degree of connectivity between patches, for each set of disturbance-colonization parameters across each of five dispersal ranges (i.e. $\delta=1,2,3,4$, or $\infty$ distance units) implemented using Eq. (3). These dispersal ranges correspond to five levels of connection between patches, ranging from nearest neighbor connections only, through to all patches being equally connected regardless of their location.

We compare the patch lifetimes, i.e. the length of time a patch is suitable, for landscapes with aggregated and random disturbances. We examine the differences between these types of disturbance across both patch spatial configurations described above $(10 \times 10$ and $2 \times 50$ grids $)$. To illustrate the relationships between the type of disturbance and the spatial configuration of patches we use the lifetimes calculated from 500 repeat simulations, evaluated using a fixed parameter set selected from within the range of that used in our other analyses. The patch lifetime comparison is done with a disturbance rate equal to 0.1 and severity equal to 0.2 (other parameters have no effect on the patch lifetimes, which are purely a consequence of habitat processes).

\subsection{Model validation}

In order to validate the simulation model we compare it with a spatially implicit, deterministic model that is an adaptation of the classic Levins (1969, 1970) metapopulation model to include habitat dynamics. It is similar to that proposed by Johnson (2000) and recently derived as the limit of a discrete-state stochastic model by Ross (2006). The habitat dynamics under this deterministic model are described by the rate of change in the amount of the suitable habitat $m$ :

$\frac{d m}{d t}=r(1-m)-d^{\prime} m$

where $r$ is the recovery rate, and $d^{\prime}=d p$ is the per patch disturbance rate. For the metapopulation dynamics, the rate of change in the proportion of patches $n$ that a species occupies is given by

$\frac{d n}{d t}=c^{\prime} n(m-n)-\left(e+d^{\prime}\right) n$,

where $c^{\prime}=c \mu_{c} / N$ is the effective colonization rate for mean number of connections per patch $\mu_{c}$, and $e$ is the intrinsic local extinction rate (not including local extinctions caused by habitat that becomes unsuitable). The equilibrium proportions $m^{*}$ of suitable and $n^{*}$ of occupied patches are then readily calculated from (5) and (6) as $m^{*}=r /\left(r+d^{\prime}\right)$ and $n^{*}=m^{*}-\left(e+d^{\prime}\right) / c^{\prime}$ (Johnson, 2000).

We compare, over a range of values for the colonization probability, the equilibrium values of the proportions of suitable and occupied patches from the deterministic model with the mean values of these proportions, conditional on persistence of the simulated metapopulation over 500 timesteps. While some differences between the models are expected, broad agreement would indicate that the simulation model is functioning properly.

\section{Results}

\subsection{Comparison of analytic and simulation results}

The stochastic simulation and deterministic analytic models show similar habitat dynamics, as represented by the mean or equilibrium number of suitable patches, although this agreement is not so close at high disturbance severities (for a fixed disturbance rate; Fig. 1a). When disturbance severity is high, many patches are disturbed at once but infrequently so, and hence the number of suitable patches varies greatly (Fig. 1b). The discrepancy between the analytic and the simulation models appears to be due to the magnitude of the variance in the number of suitable patches in the stochastic simulation.

\subsection{Correlated disturbances, colonization, and landscape configuration}

Spatially aggregated disturbances result in greater metapopulation extinction probabilities than spatially random ones across a wide range of landscape connectivities, colonization probabilities, and patch spatial configurations (Fig. 2). As expected, landscape connectivity has a strong effect on metapopulation extinction probability, with increasing connectivity resulting in large increases in persistence for a given colonization probability. At one extreme, when all patches are equally connected, there are no differences in extinction probability if we change either the type of disturbance or the patch spatial configuration; 
complete connectivity erases the impact of these spatial patterns (Fig. 2). More intriguingly when the level of connectivity decreases (from a dispersal range of 4 to a dispersal range of 1 distance unit) all of these results are strongly affected by the spatial configuration of patches (Fig. 2a and b) and the spatial pattern of disturbance (Fig. $2 \mathrm{c}$ and $\mathrm{d}$ ). For instance, the difference between simulations with spatially aggregated and random disturbances is much larger when the landscape is an elongated $2 \times 50$ grid (Fig. $2 b$ ), as opposed to a more compact square $10 \times 10$ grid (Fig. 2a). The metapopulation extinction probability can be twice as high for an elongated configuration as it is for a compact configuration for the same connectivity and total number of disturbances. The impact of the spatial configuration of the patches in the metapopulation (Fig. $2 \mathrm{c}$ and $\mathrm{d}$ ) is even stronger than the impact of the spatial pattern of the disturbance (Fig. 2a and b). Indeed, under the same disturbance regimes, it is possible for a compact metapopulation to have an extinction probability close to zero over the time-scale of the simulations, while a metapopulation with an elongated spatial configuration is almost certain to become extinct. The boundary conditions on extinction in the metapopulation system, i.e. the values of the colonization probability at which we observe either at least one persistent metapopulation or observe that all of the runs persist, summarize the substantial differences between the spatially aggregated and random disturbance regimes (Fig. 3). This difference is largest for the maximum colonization probability (i.e. the colonization rate at which at least one extinction is observed) when landscape connectivity is lowest $(\delta=1)$. As landscape connectivity increases, the colonization probability at which extinctions are observed decreases towards 0 , and the effect of spatial aggregation in disturbance also decreases. Again, the spatial configuration of patches has a strong effect on this pattern, with larger differences between correlated and random disturbances in a landscape with an elongated configuration of patches.

\subsection{Correlated disturbances, habitat configuration and patch lifetime}

Patch lifetimes, the time between recovery of a patch and its next disturbance, are strongly affected by the disturbance regime (Table 1, Fig. 4a and b). When disturbances are spatially random, the patch lifetime does not differ significantly between patches, while spatial correlation in disturbance substantially increases the variance in patch lifetime across the landscape, resulting in longer maximum and shorter minimum lifetimes (Figs. 4a and b;
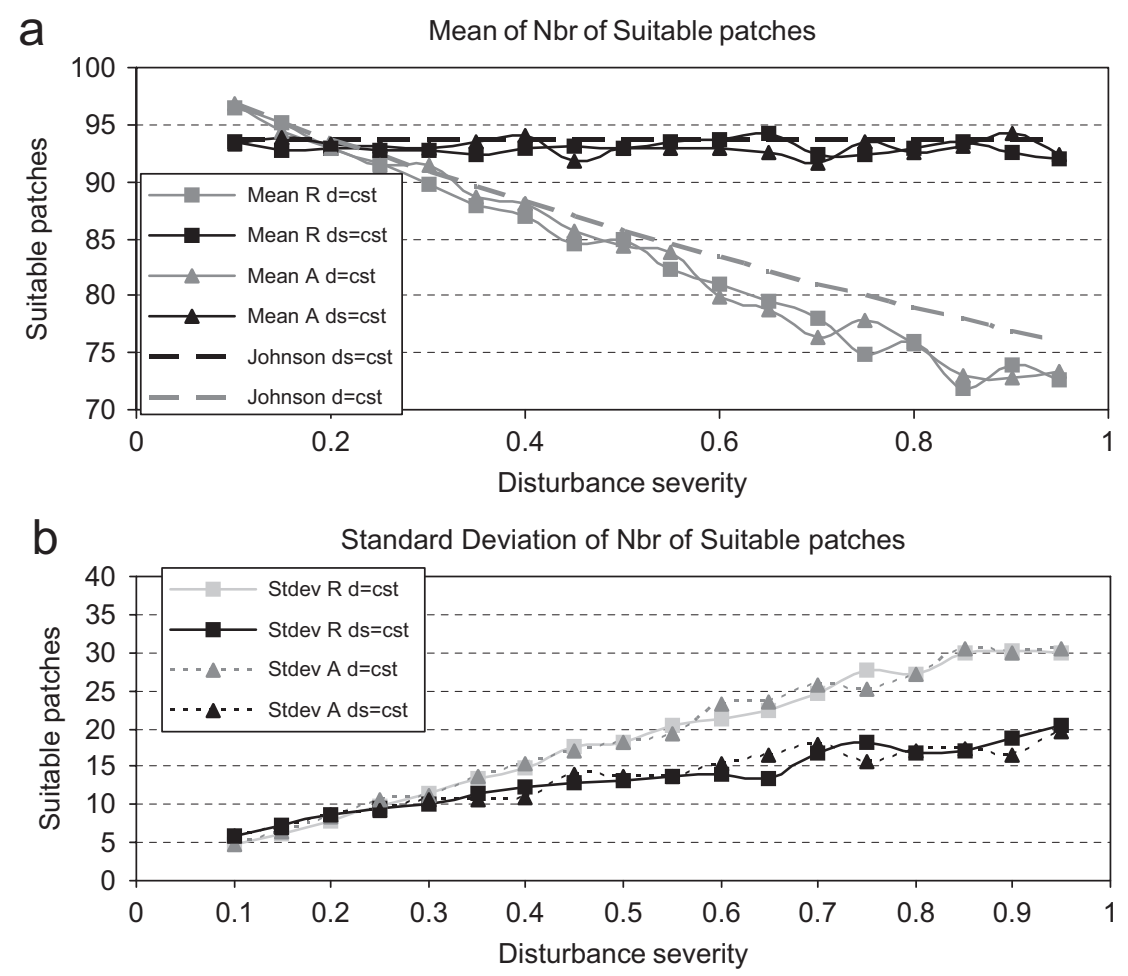

Fig. 1. (a, b). Means of the number of suitable patches (a) and standard deviation (b) at equilibrium for the model of Johnson (2000) (dashed lines), and at time 500 for our spatially explicit model (solid lines) when the recovery rate is equal to 0.3 . Here we ignore the metapopulation dynamics, including whether the population still persists at the end of the simulation, and only consider the number of suitable patches. In the Johnson model, the product $d p$ is represented as a single parameter, so here we either hold $d p=0.02$ constant (black lines), or hold $d=0.01$ constant (gray lines), and in either case vary the severity $p$. As we anticipate, given the similarities in the mechanisms of the two models, the simulation model broadly agrees with the analytical model, except at the extreme when disturbance severity is large, and particularly in the case where $d$ is constant (rather than $d s$ ). When disturbance severity is large, so is the standard deviation of the number of suitable patches (b); again, this is most evident in the constant $d$ case. 
Table 1). This is a consequence of both the disturbance regime and the spatial configuration (Fig. 4). When disturbance is correlated, patches at the edge of the landscape receive relatively fewer disturbances (Fig. 4a), while in the case of random disturbance there is little or no difference, as should be anticipated. This pattern is strongly influenced by patch spatial configuration (Fig. 4c and d). If disturbances are spatially aggregated, patches at the edge of an elongated landscape have much longer lifetimes than edge patches in a more compact landscape. But, the patches near the edge of the compact landscape are better connected to the whole system. Thus, while the most isolated patches of the elongated system might serve as effective disturbance refugia, they are poorly connected and hence have limited ability to recolonize the rest of system.

\section{Discussion}

By including habitat dynamics in spatially explicit metapopulation models (Fahrig, 1992; Gyllenberg and Hanski, 1997; Brachet et al., 1999; Johnson, 2000; Keymer et al., 2000; Amarasekare and Possingham, 2001; Ellner and Fussmann, 2003; Thomas and Hanski, 2004), it is possible to provide management and conservation plans for species in successional systems and systems subject to occasional disturbance (Possingham et al., 1994; Lindenmayer and Possingham, 1996; McCarthy and Lindenmayer, 2000; Amarasekare and Possingham, 2001; Akç akaya et al., 2004). In this paper, we use a metapopulation and patch dynamic simulation model using object-oriented programming to look closely at the implications of spatial and temporal structure of disturbances in metapopulations with different spatial patch configurations.

In general, we found that both the spatial patterns of disturbance and patch configuration affect metapopulation persistence. In our simulations, increased spatial correlation in habitat disturbance always increases the extinction probability. Population extinction is much smaller when the spatial configuration of the metapopulation is compact rather than elongated. The strength of these effects depends on the degree of connectivity amongst the patches. These results confirm the importance for metapopulation persistence of individual dispersal distance (Hanski, 1999; Hanski and Ovaskainen, 2000; Johst et al., 2002) as well as the disturbance regimes (Fahrig, 1992; Johst and Drechsler, 2003). In addition, we show that the spatial configuration could make the difference between a high probability of persistence and a high probability of extinction for a metapopulation. Under a fixed disturbance regime, a metapopulation with an elongated spatial configuration is almost certain to become extinct when compact one is predicted to remain viable in almost all cases. Such a result is due to large differences in patch lifetime that depends on the spatial location of patches. Consequently, the position of patches relative to each other in such dynamic systems could be used to help determine appropriate management strategies on a patch-specific level.

With appropriate simplifications, our simulation model generally agrees with analytic results from a spatially implicit deterministic model which includes habitat dynamics. We found a fairly close fit. We also compared the mean proportion of patches occupied in the simulation model with the value predicted at equilibrium in the analytical model. Again, when we conditioned the simulation on non-extinction, we found the patterns generated by the models generally agreed. However, there is some deviation between the two models, which is particularly apparent at low colonization rates. This difference is due to the discretization of the timing of the events. In the analytic model, the size of the population changes instantaneously and continuously, while in the stochastic simulations, colonization is discrete in size and in timing. Thus, the deviations from the analytic model, which are particularly evident when the colonization rate is low, suggest a colonization rate at which the stochasticity in the colonization process becomes important.

We looked at the impact of the pattern of disturbance on metapopulation persistence, and how this interacts with colonization rate, patch connectivity and the spatial configuration of patches. While spatial correlation in patch disturbance always results in increased extinction probabilities, these differences in metapopulation extinction depend nonlinearly on patch connectivity. When the connectivity in the system is low (dispersal only between adjoining patches), the value of the colonization rate required to achieve a given metapopulation extinction probability increased strongly as the level of connectivity decreased. Conversely, when the system is already well connected a large increase in the average number of connections per patch does not significantly increase the viability of the system (Fig. 3). The impact of an increase in the degree of patch connectivity (the number of connections per patch) also depends on the spatial configuration of patches. Indeed, for a given set of disturbance parameters, more connected patches are needed to maintain a viable metapopulation when the patch configuration is elongated. These results can be understood by looking at patch lifetimes in the various cases (Keymer et al., 2000; Frank and Wissel, 2002; Ellner and Fussmann, 2003; Hastings, 2003; Frank, 2005). According to Johst and Drechsler (2003) and Kallimanis et al. (2005), spatial correlation in patch destruction causes increasing temporal fluctuations and is generally disadvantageous for long-term persistence. Our simulation results agreed with these results: while average patch lifetimes are fairly equivalent for the spatially aggregated and random disturbance scenarios, in the aggregated case the variance in patch lifetimes is much greater and metapopulation persistence declines. Importantly, the distribution of patch lifetimes also has a distinct spatial configuration. Patches at the edges of the landscape are suitable for much longer than average lifetimes, while centrally located patches have 

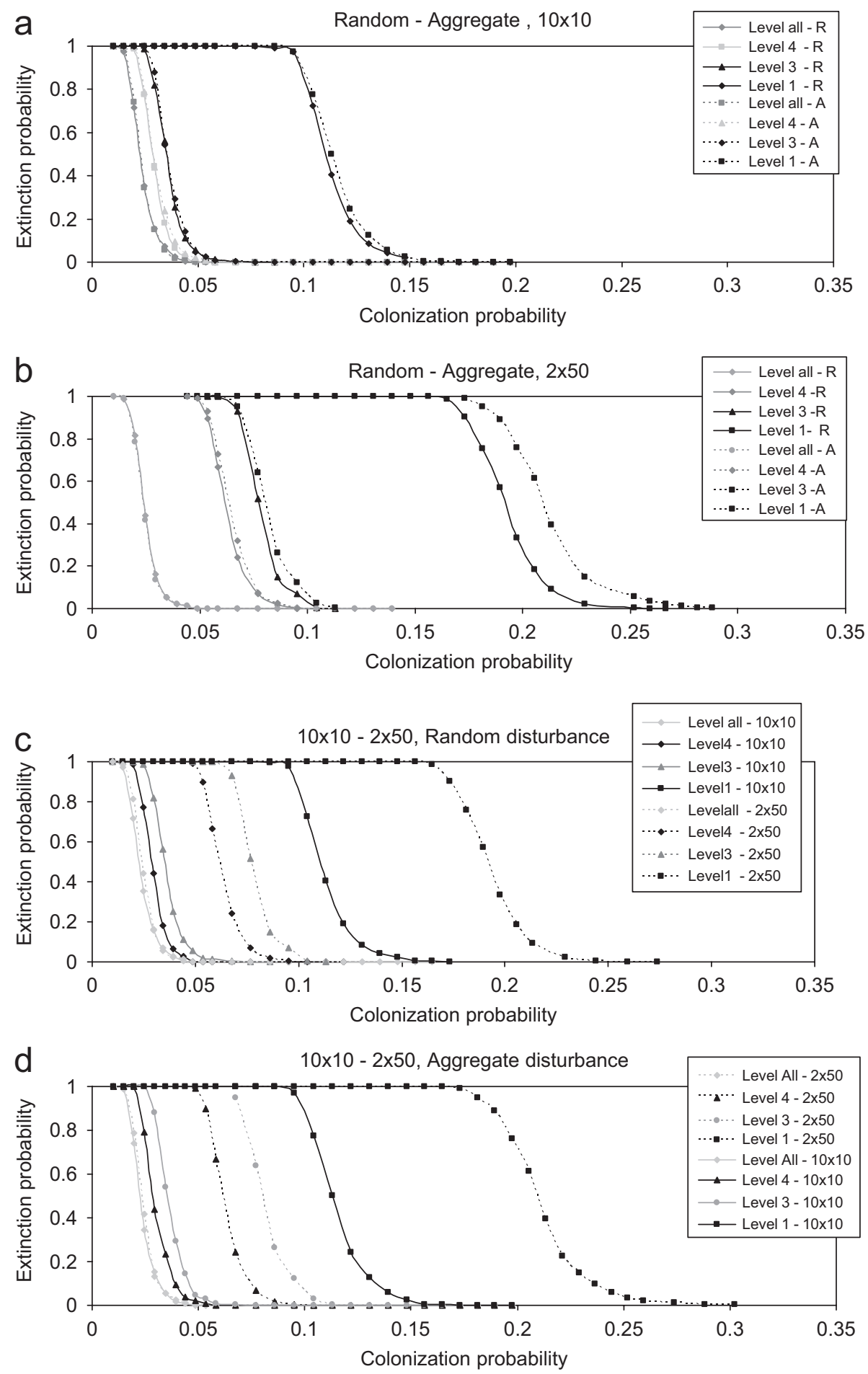

Fig 2. (a-d). Extinction probability at time 500 with varying colonization rates and for four levels of connection, in the cases where disturbance is random or spatially aggregated, with 100 habitat patches on a well-connected $10 \times 10$ grid and on a more poorly connected $2 \times 50$ grid. The rates used for the simulations are: extinction rate $=0.2$; recovery $=0.3$, disturbance rate $=0.1$ and disturbance severity $=0.2$. The level of connection defines the connectivity between habitat patches, a level of 1 means colonization is possible only between patches within a distance unit 1 , and so on. Level 5 corresponds to the case where all patches in each system are equally connected. The results are presented in two different ways to highlight the effects of both random versus spatially aggregated disturbances, (a) and (b), and the spatial structure of the patch system, (c) and (d).

much shorter ones. The implication is that the patches that are most stable, and thus most likely to be occupied, will be the ones with the fewest connections to other patches. A further implication of this pattern is that the central portion of the landscape acts as a barrier to percolation of colonization events through the landscape, as many of the patches in that area will be disturbed at a given time, and colonists landing in any suitable patch are likely to go 

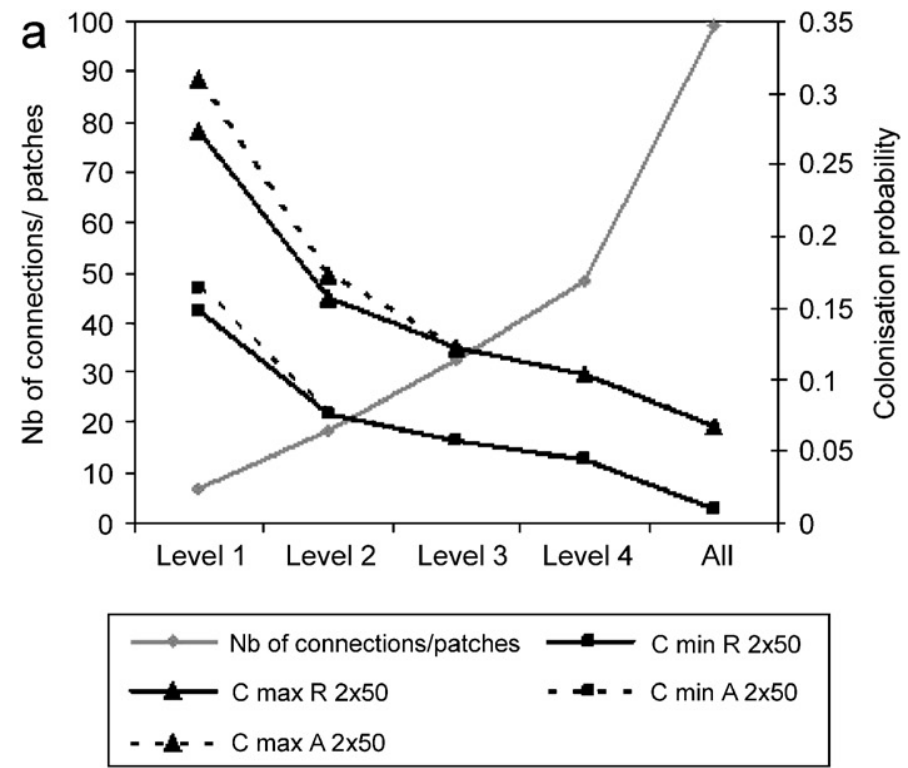
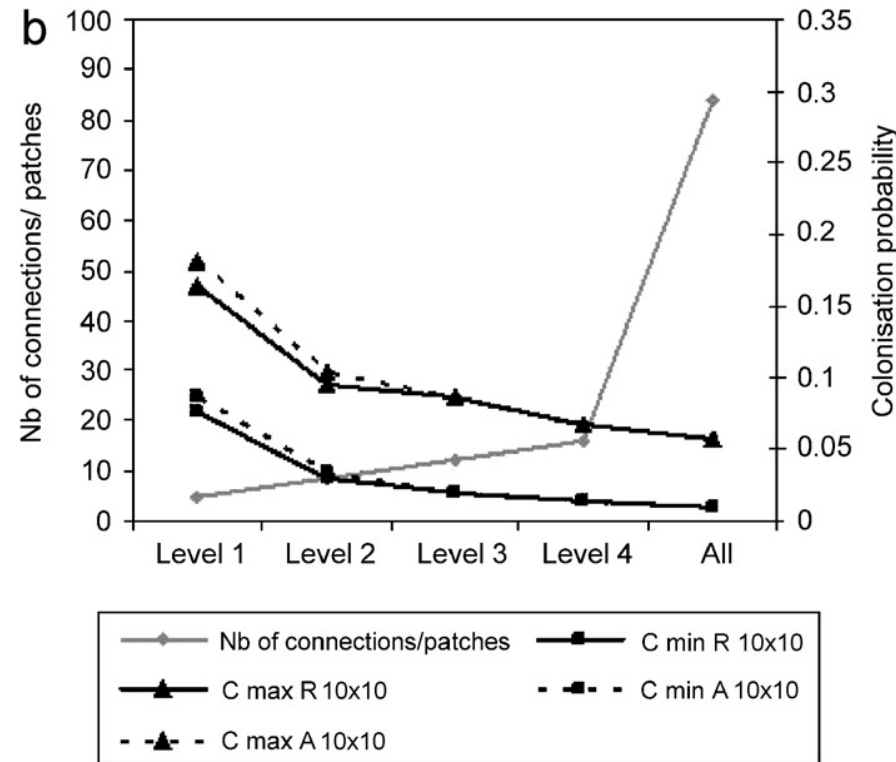

Fig. 3. Minimum and maximum value of colonization rates necessary for metapopulation persistence at different levels of connectivity, with two spatial configurations, (a) $2 \times 50$, and (b) $10 \times 10$, when disturbance is either aggregated $(A)$ or randomized $(R)$. The left abscissas and the solid squares lines show the number of connections per patch for each level of connectivity, representing the maximum distance between patches for which colonization is possible. The right abscissas and remaining lines indicate the minimum colonization rate for which metapopulations persist (square) and maximum rate for which extinctions occur (triangle) for each level of connectivity and for random (solid line) and aggregate (dashed line) disturbances.

Table 1

Patch lifetimes for different simulation scenarios

\begin{tabular}{lcclll}
\hline Patch lifetime & \multicolumn{2}{l}{$10 \times 10$} & & \multicolumn{2}{l}{$2 \times 50$} \\
\cline { 2 - 3 } \cline { 6 - 6 } \cline { 5 - 6 } & Random & Aggregated & & Random & Aggregated \\
\hline Minimum & 41.8 & 31.7 & & 39.8 & 31.5 \\
Maximum & 52.8 & 81.7 & & 53.6 & 78.3 \\
Mean & 46.3 & 46.1 & & 46.9 & 41.1 \\
Standard deviation & 2.0 & 11.3 & & 2.2 & 8.7 \\
\hline
\end{tabular}

Statistics for patch lifetimes are calculated from 45000 observations for each scenario.

extinct quickly due to the frequent disturbances. In the system we have considered, patches on the periphery of a system present a double-bind: they are more stable in the face of correlated disturbance yet less suitable as sources of colonists. The effectiveness of edge patches as potential refugia may therefore vary greatly, according to whether their stability or isolation proves the dominant factor. This further highlights the importance for conservation management of careful assessments of the relative contributions of edge versus interior patches to persistence (Sutherland et al., 2006).

The elongated patch configuration illustrates the interaction colonization probability and disturbance probabilities. Here the minimum patch lifetime is similar to that for the $10 \times 10$ case, however, the maximum, mean, and variance are all lower. This difference is driven by the lower "concentration" of patches. That is, the spatially aggregated disturbances are spread more evenly over the landscape in the $2 \times 50$ case, as can be seen in Fig. 4, with the exception of the patches at the far ends of the landscape. Thus, for a given parameter set this system has a higher probability of extinction, or conversely requires a higher colonization rate to realize the same extinction rate, compared with the more compact $10 \times 10$ case. The explanation is that not only are patch lifetimes on average shorter, but also the very persistent patches are quite far apart, and colonization must percolate through a very large set of patches each of which has a relatively short lifetime.

The patterns in patch lifetime also suggest why patch connectivity plays such a significant role in determining when spatially aggregated disturbances produce different results from random ones. If reasonably distant patches are connected, then colonists can move between patches that are quite stable, thus the dynamics of the system will be driven primarily by the colonization dynamics of these patches (and population extinction). In an extreme case, such a system may become equivalent to a system with fewer patches, but where there are no habitat dynamics.

Management that decreases spatial correlation in disturbance will have relatively little effect in compact systems, in comparison with less compact systems. In either case, large gains in metapopulation persistence can be made if patch connectivity is low and can be increased. In contrast, when patch connectivity is high it is the most effective strategy is to increase colonization rates. This difference in management actions has implications that may vary across landscape. Low connectivity suggests more corridors should be added; once patches are reasonably well-connected, effort should focus on facilitating movement through those corridors, i.e. increasing the 


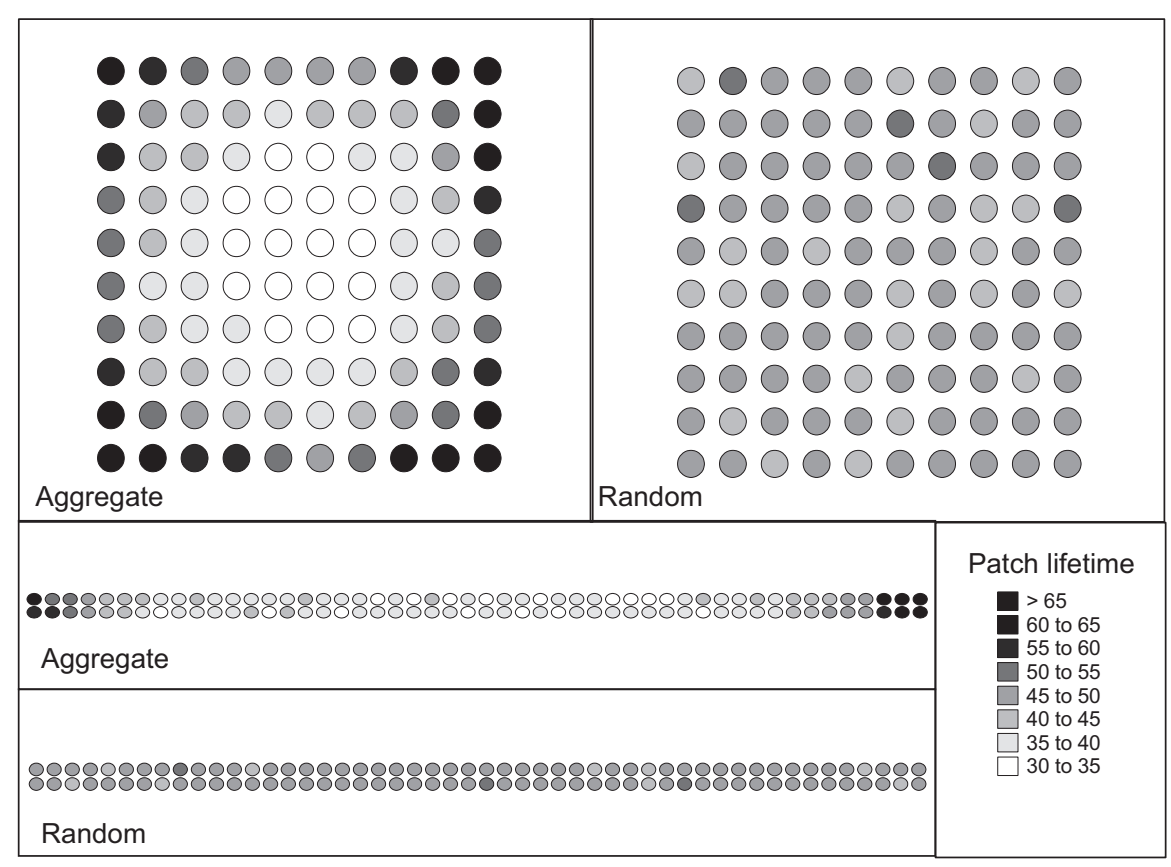

Fig. 4. Spatial patterns of mean patch lifetimes for random and aggregate disturbances on systems of habitat patches on a $10 \times 10$ grid and the $2 \times 50$ system of patches. In each case, the disturbance rate is equal to 0.1 while the disturbance severity is 0.2 .

colonization rate. However, in the less compact case, if dispersal is limited, reasonably large gains can also be made if spatial correlation in disturbances can be reduced. In many types of habitat, disturbance may be state dependent, for instance the frequency and intensity of fire in a patch depends on the state of vegetation in the patch (Cochrane, 2003), and thus management of disturbances can be achieved by management of habitat.

Much remains to be learnt about the consequences of the interplay between spatially complex metapopulation and habitat processes, but our results go some way to exploring these and have significant implications for the spatial management of ecological systems. While demonstrating that assemblages of individuals of a species distributed across a patchy habitat function as a metapopulation has often proven difficult (Harrison, 1991), many species and ecological systems are now managed based on the assumption that they function as a metapopulation (Hanski, 1999; Hanski and Ovaskainen, 2000; Frank and Wissel, 1998, 2002). Accepting the premise of metapopulation dynamics, our results show that the spatial structure of habitat dynamics plays a central role in determining persistence, and defining the most effective management tools. If disturbances are either spatially correlated, for instance the effects of hurricanes or droughts on habitat, or are contagious, such as forest fires, patches at the edge of the landscape may serve as important stable sites that support the metapopulation during disturbance events. The value of these patches must be balanced against the negative impacts that they may suffer due to anthropogenic influences or other edge-related phenomena and their limited ability to recolonize the remainder of this system.

\section{Acknowledgments}

The work of B.J.C. was supported by a Ph.D. scholarship from the Australian Research Council Centre of Excellence for Mathematics and Statistics of Complex Systems.

\section{References}

Akçakaya, H.R., Volker, V.C., Radeloff, D.J., Mladenoff, He, H.S., 2004. Integrating landscape and metapopulation modeling approaches: viability of the sharp-tailed grouse in a dynamic landscape. Conserv. Biol. 18, 526-537.

Amarasekare, P., Possingham, H.P., 2001. Patch dynamics and metapopulation theory: the case of successional species. J. Theor. Biol. 209, 333-344.

Brachet, S., Olivieri, I., Godelle, I., Klein, E., Frascaria-Lacoste, N., Gouyon, P.H., 1999. Dispersal and metapopulation viability in a heterogeneous landscape. J. Theor. Biol. 198, 479-495.

Cochrane, M.A., 2003. Fire science for rainforest. Nature 421, 913-919.

Diffendorfer, J.E., Gaines, M.S., Holt, R.D., 1999. Patterns and impacts of movements at different scales in small mammals. In: Barrett, G.W., Peles, J.D. (Eds.), Landscape Ecology of Small Mammals. Springer, New York, pp. 63-88.

Doak, D.F., Marino, P., Kareiva., P.M., 1992. Spatial scale mediates in influence of habitat fragmentation on dispersal success: implications for conservation. Theor. Popul. Biol. 41, 315-336.

Doebeli, M., Ruxton, G.D., 1998. Stabilization through spatial pattern formation in metapopulations with long-range dispersal. Proc. R. Soc. London Ser. B 265, 1325-1332.

Downing, K., Reed, M., 1996. Object-oriented migration modelling for biological impact assessment. Ecol. Modelling 93, 203-219.

Ellner, S.P., Fussmann, G., 2003. Effects of successional dynamics on metapopulation persistence. Ecology 84, 882-889.

Fahrig, L., 1992. Relative importance of spatial and temporal scales in a patchy environment. Theor. Popul. Biol. 41, 300-314. 
Fahrig, L., Merriam, G., 1985. Habitat patch connectivity and population survival. Ecology 66, 1762-1768.

Frank, A., 2005. Metapopulation persistence in heterogeneous landscapes: lessons about the effect of stochasticity. Am. Nat. 165, 374-388.

Frank, K., Wissel, C., 1998. Spatial aspects of metapopulation survivalfrom model results to rules of thumb for landscape management. Land Ecol. 13, 363-379.

Frank, K., Wissel, C., 2002. A formula for the mean lifetime of metapopulations in heterogeneous landscapes. Am. Nat. 159, 530-552.

Fryxell, J.M., 2001. Habitat suitability and source-sink dynamics of beavers. J. Anim. Ecol. 70, 310-316.

Gurevitch, J., Padilla, D.K., 2004. Are invasive species a major cause of extinctions? TREE 19, 470-474.

Gyllenberg, M., Hanski, I., 1997. Habitat deterioration, habitat destruction, and metapopulation persistence in a heterogenous landscape. Theor. Popul. Biol. 52, 198-215.

Hanski, I., 1999. Metapopulation Ecology. Oxford University Press, Oxford.

Hanski, I., Ovaskainen, O., 2000. The metapopulation capacity of a fragmented landscape. Nature 404, 755-758.

Hansson, L., 1991. Dispersal and connectivity in metapopulations. Biol. J. Linn. Soc. 42, 89-103.

Harrison, S., 1991. Local extinction in a metapopulation context: an empirical evaluation. Biol. J. Linn. Soc. 42, 73-88.

Hastings, A., 2003. Metapopulation persistence with age-dependent disturbance or succession. Science 301, 1525-1526.

Johnson, M.P., 2000. The influence of patch demographics on metapopulations, with particular reference to successional landscape. Oikos $88,67-74$.

Johst, K., Drechsler, M., 2003. Are spatially correlated or uncorrelated disturbance regimes better for the survival of species. Oikos 103, 449-456.

Johst, K., Brandl, R., Eber, S., 2002. Metapopulation persistence in dynamic landscape: the role of dispersal distance. Oikos 98, 263-270.

Kallimanis, A.S., Kunin, W.E., Halley, J.M., Sgardelis, S.P., 2005. Metapopulation extinction risk under spatially autocorrelated disturbance. Conserv. Biol. 19, 534-546.

Keymer, J.E., Marquet, P.A., Velasco-Hernandez, J.X., Levin, S.A., 2000. Extinction thresholds and metapopulation persistence in dynamic landscapes. Am. Nat. 156, 478-494.

Levins, R., 1969. Some demographic and genetic consequences of environmental heterogeneity or biological control. Bul. Ent. Soc. Am. 68, 237-240.
Levins, R., 1970. Extinction, Lecture Notes in Mathematics, vol. 2. Springer, Berlin, pp. 75-107.

Lindenmayer, D., Possingham, H., 1996. Ranking conservation and timber management options for Leadbetter's possum in Southeastern Australia using population viability analysis. Conserv. Biol. 10, 235-251.

Manly, B.F.J., 1991. Randomization and Monte Carlo Methods in Biology. Chapman \& Hall, London, 292p.

McCarthy, A., Lindenmayer, D., 2000. Spatially-correlated extinction in a metapopulation model of Leadbeater's Possum. Biodiversity Conserv. 9, 47-63.

Moilanen, A., Hanski, I., 2001. On the use of connectivity measures in spatial ecology. Oikos 95, 147-151.

Possingham, H., Lindenmayer, D., Norton, T.W., 1994. Metapopulation viability analysis of the greater glider Petauroides volans in a wood production area. Biol. Conserv. 70, 227-236.

Ross, J.V., 2006. A stochastic metapopulation model accounting for habitat dynamics. J. Math. Biol. 52, 788-806.

Sutherland, W.J., Armstrong-Brown, S., et al., 2006. The identification of 100 ecological questions of high policy relevance in the UK. J. Appl. Ecol. 43, 617-627.

Thomas, C.D., 1994. Extinction, colonization and metapopulations: environmental tracking by rare species. Conserv. Biol. 8, 373-378.

Thomas, C.D., Hanski, I., 2004. Metapopulation dynamics in changing environments: butterfly responses to habitat and climate change. In: Hanski, I., Gaggiotti, O.E. (Eds.), Ecology, Genetics and Evolution of Metapopulations. Elsevier, Amsterdam, pp. 489-514.

Thomas, J.A., Bourn, N.A.D., Clarke, R.T., Stewart, K.E., Simcox, D.J., Pearman, G.S., Curtis, R., Goodger, B., 2001. The quality and isolation of habitat patches both determine where butterflies persist in fragmented landscapes. Proc. R. Soc. London Ser. B 268, 1791-1796.

Wahlberg, N., Klemetti, T., Hanski, I., 2002. Dynamic populations in a dynamic landscape: the metapopulation structure of the marsh fritillary butterfly. Ecography 25, 224-232.

Wiens, J.A., 2001. The landscape context of dispersal. In: Clobert, E.D.J., Dhondt, A.A., Nichols, J.D. (Eds.), Dispersal. Oxford University Press, New York, pp. 96-109.

Wilcox, C., Cairns, B.J., Possingham, H.P., 2006. The role of habitat disturbance and recovery in metapopulation persistence. Ecology 87, 855-863. 